
\title{
$\angle S$ Research Square \\ Erectile Dysfunction Among Testicular Cancer Survivors: A Systematic Review and Meta-Analysis, 2020
}

Sitotaw Kerie ( $\square$ sitkere5@gmail.com )

Bahir Dar University

Yinager Workineh

Bahir Dar University

Ayele Semachew

Bahir Dar University

Emiru Ayalew

Bahir Dar University

Melak Menberu

Bahir Dar University

\section{Research Article}

Keywords: Testicular cancer, erectile dysfunction, pooled prevalence, clinical research, chronic diseases.

Posted Date: February 10th, 2021

DOI: https://doi.org/10.21203/rs.3.rs-156138/v1

License: (c) (1) This work is licensed under a Creative Commons Attribution 4.0 International License.

Read Full License 
Erectile dysfunction among testicular cancer survivors: a systematic review and metaanalysis, 2020.

Sitotaw Kerie ${ }^{1 *}$, Yinager Workineh ${ }^{2}$, Ayele Semachew $^{3}$, Emiru Ayalew $^{4}$, Melak Menberu ${ }^{5}$

${ }^{1}$ Department of Adult Health Nursing, College of Medicine and Health Sciences, Bahir Dar University, Bahir Dar, Ethiopia

${ }^{2}$ Department of Child Health Nursing, College of Medicine and Health Sciences, Bahir Dar University, Bahir Dar, Ethiopia

${ }^{3}$ Department of Adult Health Nursing, College of Medicine and Health Sciences, Bahir Dar University, Bahir Dar, Ethiopia

${ }^{4}$ Department of Adult Health Nursing, College of Medicine and Health Sciences, Bahir Dar University, Bahir Dar, Ethiopia

${ }^{5}$ Department of Psychiatry, College of Medicine and Health Sciences, Bahir Dar University, Bahir Dar, Ethiopia

*The corresponding author

Email:sitkere5@gmail.com 


\begin{abstract}
Background: Erectile dysfunction is one of the common complications of testicular cancer with the prevalence of $11.3 \%$ to $84 \%$. It has devastating effects on men and partner's quality of life, sexual satisfaction, and sexual experience. The findings of the previous studies on this matter were uneven and inconsistent. Therefore, this systematic review and meta-analysis is conducted in order to acquire a more recent and comprehensive result.
\end{abstract}

Methods and materials: To search and retained all the eligible articles different databases were searched. These includes PubMed, Scopus, Goggle scholar, Science Direct, African Index Medicus, African Journal online, EMBASE, and Cochrane Library. To extract all the relevant information, a standardized data extraction checklist was prepared. STATA 14 statistical software was utilized to analyze the data. $\mathrm{I}^{2}$ statistics was employed to check for the heterogeneity of studies. Using a funnel plot and Egger's regression test publication bias was checked. Then, a randomeffect model was computed to estimate the pooled prevalence of erectile dysfunction.

Result: Fourteen full-text studies were included in this systematic review and meta-analysis. The pooled prevalence of erectile dysfunction among testicular cancer survivors was found to be 34.60\% (95\% CI: 25. 89, 43.30\% $\left[\mathrm{I}^{2}=95.9 \%, \mathrm{p}=0.000\right]$ ). Study design subgroup analysis indicated that the pooled prevalence of erectile dysfunction was $50.02 \%$ (95\% CI: $22.78,77.28 \%$ $\left.\left[\mathrm{I}^{2}=96.1, \mathrm{p}=0.000\right]\right)$ and $27.36 . \%\left(95 \% \mathrm{CI}: 19.23,34.48 \%\left[\mathrm{I}^{2}=91.6, \mathrm{P}=0.000\right]\right)$ in the case control and cohort studies respectively. Likewise, the level of erectile dysfunction was varied based on erectile dysfunction measuring tools and testicular cancer treatment modalities.

Conclusion: The pooled prevalence of erectile dysfunction in testicular cancer survivors was found to be high. It had also a considerable discrepancy between the study designs, and measuring 
tools and treatment modalities of testicular cancer. Therefore, there is a sound to diminish the high burden of this problem.

Keywords: Testicular cancer, erectile dysfunction, pooled prevalence, clinical research, chronic diseases. 


\section{Introduction:}

Testicular cancer (TC) is a malignant tumor of the male sex organ mainly affect reproductive age groups [1]. The global incidence of testicular cancer showed a 1.80 doubling increase from 37,231 in 1990 to 66,833 new cases in 2016 [2].

Testicular cancer survivors are at greater risk of reduced sexual interest, sexual activity, sexual enjoyment, erectile dysfunction (ED), ejaculatory problems, increased sexual discomfort and changes in body image as compared with the healthy male population [3]. It has a paramount and persistent impact on a patient's sexuality due to its location and treatments [4].

Erectile dysfunction (ED), is the inability to gain or sustain an erection steady enough for sexual intercourse [5]. ED may be provoked by different factors including the adverse effects of cancer treatment, such as fatigue, pain, or anxiety related to the cancer therapy and depressed moods about the diagnosis of cancer [5].

ED can be happen in the form of organic and/or psychogenic [3]. Organic ED resulting from the effects of radiotherapy and chemotherapy, whereas psychogenic ED is associated with changes in body image, loss of sense of manliness after orchiectomy, reduced spirits of well-being and other psychosocial fluctuations associated with cancer [3].

Erectile dysfunction is one of the common complications of testicular cancer with a prevalence of $11.3 \%$ to $84 \%$, according to different studies $[3,6]$. It has devastating effects on men and partner's quality of life, sexual satisfaction, and sexual experience $[7,8]$. Its impact is not only related to the sexual life of the survivors rather than it comprises psychological, biological, relational, and cultural elements of life [9]. 
Treatment modalities, psychological emotion, relationship, body image, types of testicular cancer, patient age and degree of ED before starting cancer treatment are some of the determinants that affect the erectile function of testicular cancer survivors [1, 5, 10-15]

Nowadays the prevalence of ED is increased due to the increment of testicular cancer survivors and decrement the mortality rate of testicular cancer patients [2]. The progressively increasing number of survivors and succeeding accomplishment of primary cancer treatments, causes specific complications continue to affect cancer survivors negatively $[4,16]$.

However, the number of testicular cancer survivors is steadily increasing with decreasing mortality rate and with the increment of complications, including erectile dysfunction in the last two decades $[2,16]$. The findings of the previous studies on this matter were uneven and inconsistent. Hence, designed and applied rationalized intervention for currently existed erectile dysfunction in testicular cancer survivors by using those fragmented study findings as evidence is not acceptable.

Therefore, we decided to conduct a systematic review and meta-analysis of the existing data in order to acquire a more recent and comprehensive result. This evidence will give a new information for policy makers, which enables them to design scientific directives to decrease the magnitude of ED among testicular cancer survivors. 


\section{Methods}

\subsection{Protocol and registration}

The findings of this review were reported, according to the preferred reporting item on systematic review and meta-analysis statement [17]. It is not registered in the Prospero database.

\subsection{Eligibility criteria}

The inclusion criteria were: 1 . Any primary studies that clearly reported the prevalence of erectile dysfunction among testicular cancer survivors, 2. Studies conducted between 2001 and 2020, 3. Studies published in English, 4. Studies available at the electronic source before July 2020. On the other hand, qualitative studies, citations without complete abstract and/or full text, anonymous reports, editorials, conference presentations, letters, expert opinions, case reports, and duplications were excluded.

\subsection{Information source}

PubMed, Scopus, Goggle scholar, Science Direct, African Index Medicus, African Journal online, EMBASE, and Cochrane Library up to July 2020. Furthermore, the reference lists of related papers were also plaid to identify additional studies. And articles with incomplete data were accessed by communicating with the corresponding author.

\subsection{Searching strategy}

The main search terms and phrases were "prevalence", "magnitude", "epidemiology", “proportion", “erectile dysfunction”, “sexual dysfunction”, “impotence”, “sexual disorder", "testicular cancer" and "testicular tumor", testicular neoplasm", survivors," patients". "OR" and “AND” were used discretely and together as Boolean operators. 


\subsection{Study selection}

Duplicate articles were removed using Endnote version 7 referencing software. The title and abstract section of all the retained articles were reviewed and screened by five independent reviewers. In case of any disagreement, it was handled based on established article selection criteria.

\subsection{Data extraction}

Data was extracted by adopting the Joanna Briggs Institute (JBI) data extraction format [18]. Five authors (SK, YW, AS, EA, and MM) independently extracted all necessary data using this format. The data extraction format included primary author, publication year, country, region, measuring tool, study design, response rate, sample size, and prevalence.

\subsection{Outcome measurement}

The outcome variable of study was erectile dysfunction in testicular cancer, which is the inability to obtain or maintain an erection firm enough for sexual intercourse was measured by different tools [5]. The pooled prevalence was calculated by dividing the total number of erectile dysfunction in all review studies to the total number of involved testicular cancer survivors in the study and multiplying by 100 [19]. Erectile dysfunction $=($ Number of erectile dysfunction/number of participants) $* 100$.

\subsection{Quality assessment}

The Newcastle-Ottawa Quality Assessment tool was used to check the quality of studies in this review [20]. The assessment tool contains 1) representativeness of the sample, 2) sample size, 3) non-respondents and 4) ascertainment of the exposure, 5) independent blind assessment and 6) 
statistical test). Finally, based on this tool, article with a scale of 6 out of 10 was considered as good quality.

Each original study was evaluated by five authors independently using this tool. If there were disagreements between those five authors, the consensus was reached by taking the mean score of the five authors.

\section{Statistical analysis}

Publication bias was checked by funnel plot and more objectively through Begg's and Egger's regression test [21]. Heterogeneity of studies was quantified using the I-Squared Statistic, in which 25,50 , and $75 \%$ represented low, moderate and high heterogeneity respectively [22, 23]. Pooled analysis was conducted using a weighted inverse variance random-effects model [24]. Subgroup analysis was done by treatment modalities, and erectile dysfunction measuring tools. Sensitivity analysis was employed to see the effect of single study on the overall estimation. STATA version 14 statistical software was used for meta-analysis. 


\section{Results}

\subsection{Characteristics of reviewed studies}

Originally, 82 records were collected in relation to erectile dysfunction in testicular cancer survivors PubMed, Google scholar, Africa Index Medicus, Africa Journal Online, EMBASE, and Science Direct databases. Twenty studies were investigated from other sources. From these, 70 were not considered for further evaluation as a result of duplication and title and abstract did not appropriate search criteria. From the rest 32 articles, 11 were excluded because there were out of interest. Therefore, 21 full-text articles were retrieved and evaluated for eligibility based on the inclusion criteria. Seven articles were excluded as a result of not fulfilling our inclusion criteria [1, $3,4,6,10,12,14,15,25-37]$. Finally, 14 studies that fulfilled the eligibility criteria were included in the systematic review and meta-analysis (Figure 1).

Data for the fourteen eligible studies were extracted and analyzed in this study. The pooled prevalence of erectile dysfunction was estimated by using 7043 testicular cancer survivors. 

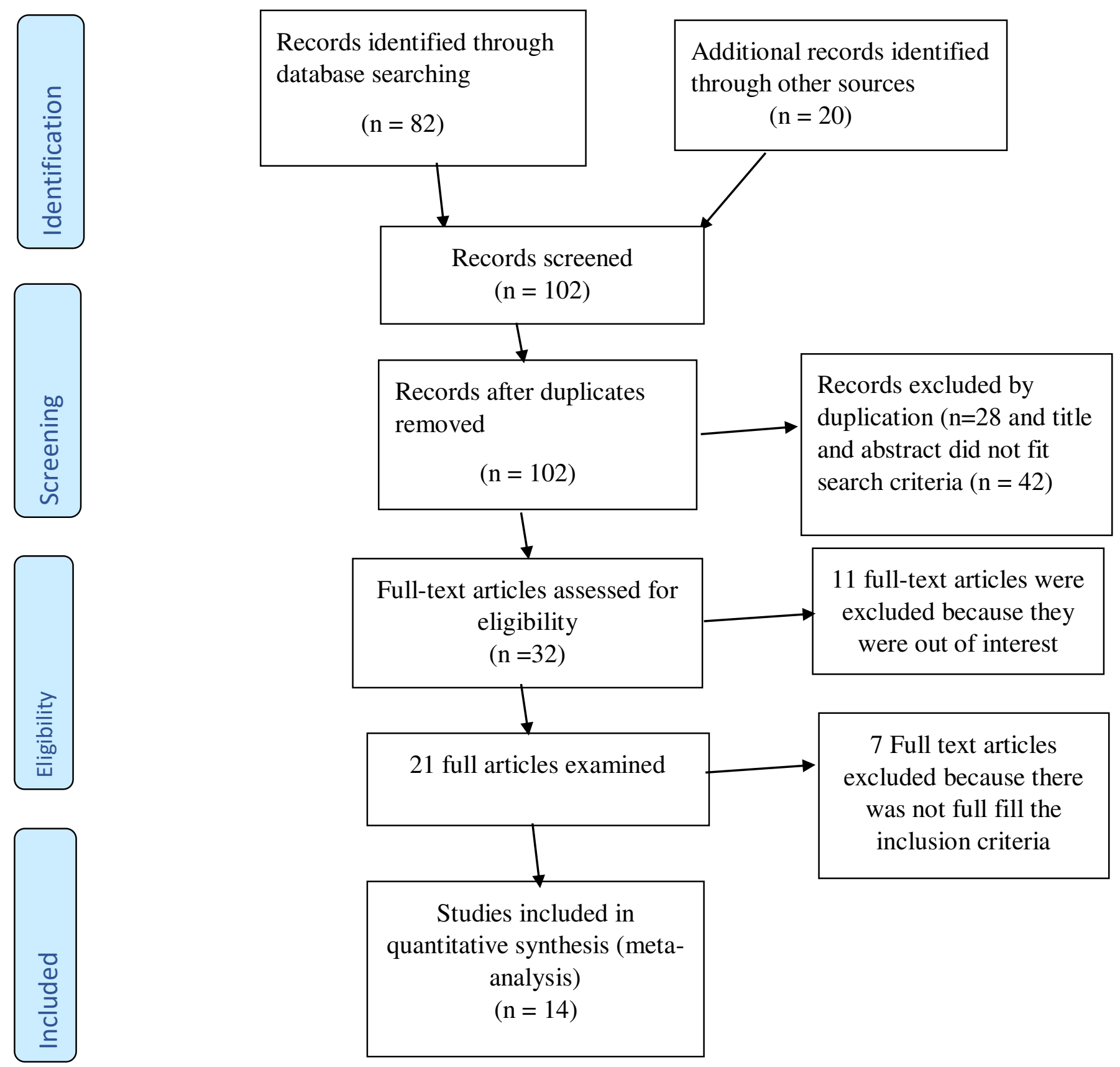

Figure 1. Flowchart to a selection of studies for a systematic review and meta-analysis of the prevalence of erectile dysfunction, 2020. 


\subsubsection{Quality appraisal}

The Newcastle-Ottawa Scale quality appraisal criteria was used. The studies included in this systematic review and meta-analysis had no low quality. Therefore, all fourteen studies were included (Table 1).

Table 1: Scoring of the quality of articles by authors using The Newcastle-Ottawa Quality Assessment tool.

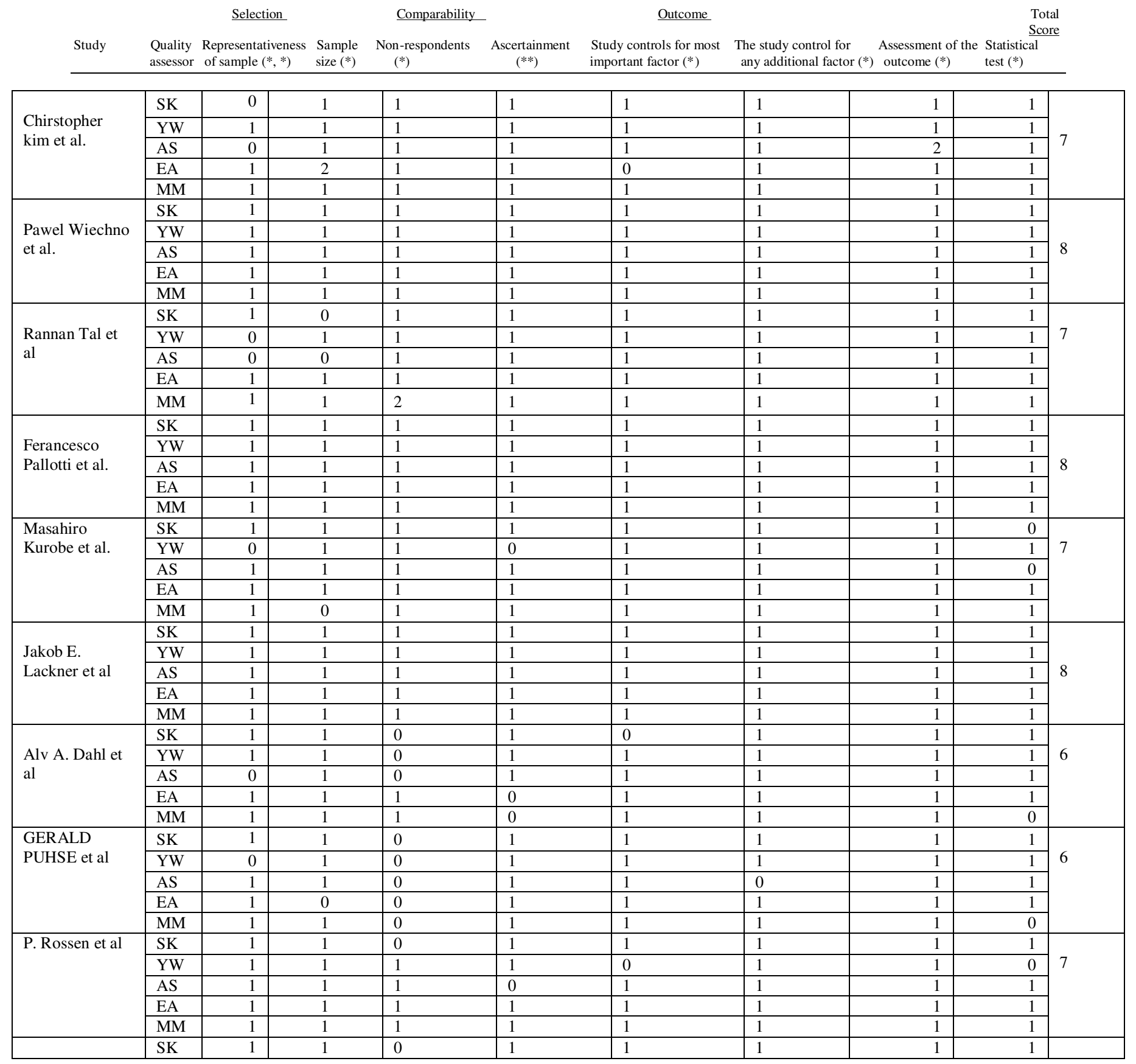




\begin{tabular}{|c|c|c|c|c|c|c|c|c|c|c|}
\hline \multirow{4}{*}{$\begin{array}{l}\text { Uros } \\
\text { Bumbasirevic } \\
\text { et al }\end{array}$} & YW & 1 & 1 & 1 & 1 & 1 & 1 & 1 & 1 & \multirow{4}{*}{7} \\
\hline & AS & 1 & 1 & 0 & 1 & 1 & 1 & 1 & 1 & \\
\hline & EA & 1 & 1 & 0 & 1 & 1 & 1 & 1 & 1 & \\
\hline & MM & 1 & 1 & 1 & 1 & 1 & 1 & 1 & 1 & \\
\hline \multirow{5}{*}{$\begin{array}{l}\text { K. } \\
\text { Dimitropoulos } \\
\text { et al }\end{array}$} & SK & 1 & 0 & 1 & 1 & 1 & 1 & 1 & 1 & \multirow{5}{*}{7} \\
\hline & YW & 1 & 1 & 1 & 1 & 1 & 1 & 1 & 1 & \\
\hline & AS & 1 & 1 & 1 & 0 & 1 & 1 & 1 & 1 & \\
\hline & EA & 1 & 1 & 1 & 1 & 1 & 1 & 1 & 0 & \\
\hline & MM & 1 & 1 & 1 & 1 & 1 & 1 & 1 & 0 & \\
\hline \multirow{5}{*}{$\begin{array}{l}\text { Paolo } \\
\text { Capogrosso et } \\
\text { al }\end{array}$} & SK & 1 & 0 & 0 & 1 & 1 & 1 & 1 & 1 & \multirow{5}{*}{6} \\
\hline & YW & 1 & 1 & 1 & 1 & 1 & 1 & 1 & 0 & \\
\hline & AS & 1 & 0 & 1 & 1 & 1 & 1 & 0 & 1 & \\
\hline & EA & 1 & 0 & 1 & 1 & 1 & 1 & 1 & 1 & \\
\hline & MM & 1 & 1 & 0 & 1 & 1 & 1 & 1 & 0 & \\
\hline \multirow{5}{*}{$\begin{array}{l}\text { Mikkel bandak } \\
\text { et al }\end{array}$} & SK & 1 & 1 & 1 & 1 & 1 & 1 & 1 & 1 & \multirow{5}{*}{8} \\
\hline & YW & 1 & 1 & 1 & 1 & 1 & 1 & 1 & 1 & \\
\hline & AS & 1 & 1 & 1 & 1 & 1 & 1 & 1 & 1 & \\
\hline & EA & 1 & 1 & 1 & 1 & 1 & 1 & 1 & 1 & \\
\hline & MM & 1 & 1 & 1 & 1 & 1 & 1 & 1 & 1 & \\
\hline \multirow{5}{*}{$\begin{array}{l}\text { Sarah L. Kerns, } \\
\text { et al }\end{array}$} & SK & 1 & 1 & 1 & 1 & 1 & 1 & 1 & 1 & \multirow[t]{5}{*}{8} \\
\hline & YW & 1 & 1 & 1 & 1 & 1 & 1 & 1 & 1 & \\
\hline & AS & 1 & 1 & 1 & 1 & 1 & 1 & 1 & 1 & \\
\hline & EA & 1 & 1 & 1 & 1 & 1 & 1 & 1 & 1 & \\
\hline & MM & 1 & 1 & 1 & 1 & 1 & 1 & 1 & 1 & \\
\hline
\end{tabular}

\subsection{Meta-analysis}

\subsubsection{Prevalence of erectile dysfunction}

In this study, the pooled prevalence of erectile dysfunction among testicular cancer survivors was found to be $34.60 \%$ (95\% CI: $25.89,43.30 \%$ ). Severe heterogeneity was detected across the studies $\left(\mathrm{I}^{2}=95.9 \%, \mathrm{p}=0.000\right)$ (Figure 2). 


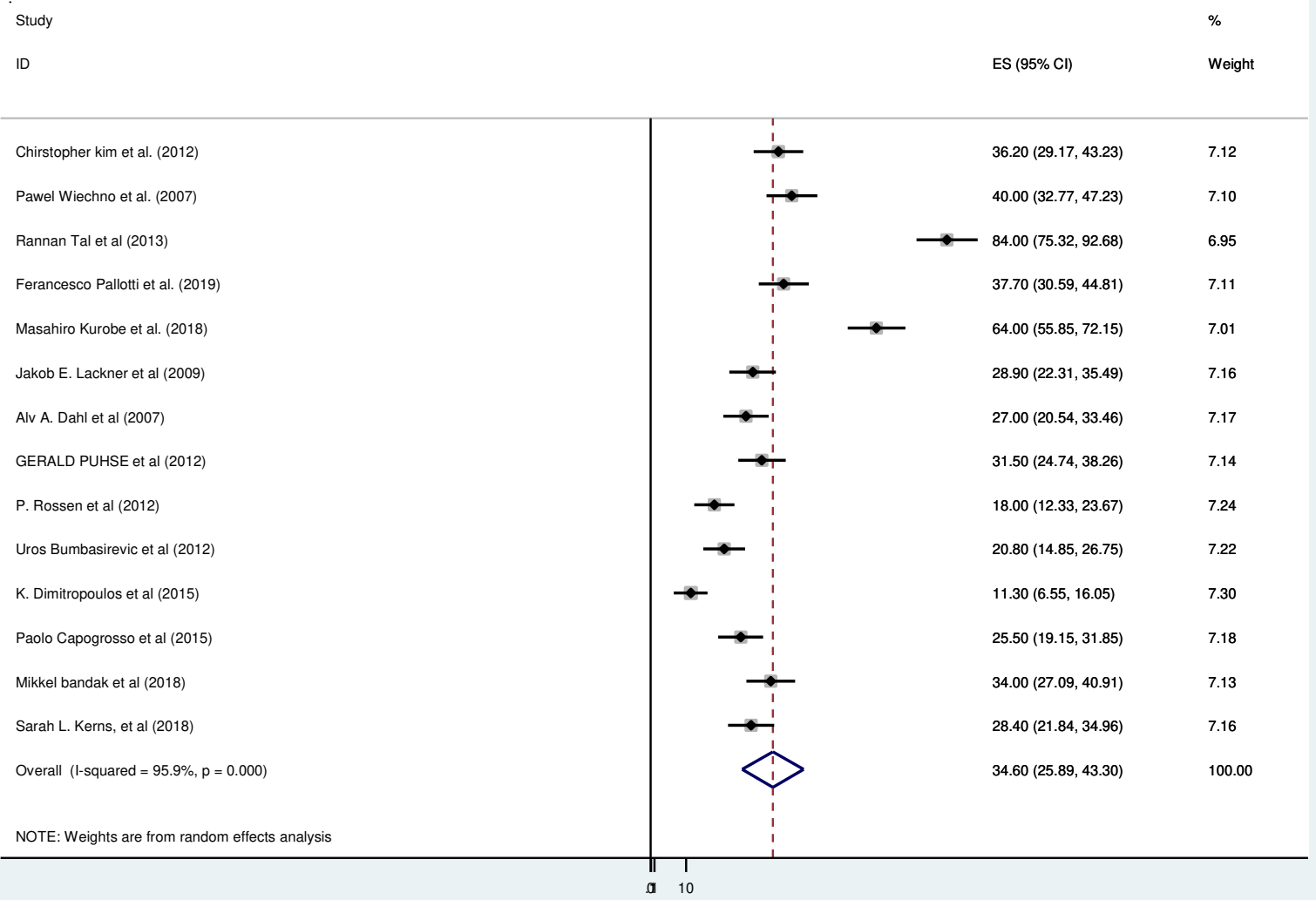

Figure 2. Pooled prevalence of erectile dysfunction among testicular cancer survivors, $2020(n=14)$.

\subsubsection{Subgroup analysis}

After the heterogeneity statistics has been done, we carried out subgroup analysis based on the study design, treatment and measuring tools. In this regard, the prevalence was higher in the case control study, 50.02\% (95\% CI: 22.78, 77.28\% [ $\left.\left.\mathrm{I}^{2}=96.1, \mathrm{p}=0.000\right]\right)$ as compared with cohort study, 27.36. \% (95\% CI: 19.23, 34.48\% $\left.\left[\mathrm{I}^{2}=91.6, \mathrm{P}=0.000\right]\right)$. The prevalence of erectile dysfunction measured by IIEF was higher than the prevalence measured by other measuring tools. The prevalence of erectile dysfunction among those who were treated with three treatment modalities (radiation, surgery and chemotherapy) was also higher than those who were treated with less than three treatment modalities (Table 2). 
Table 2. Subgroup analysis of prevalence of erectile dysfunction among testicular cancer survivors, 2020.

\begin{tabular}{llll}
\hline Variables & Characteristics & Estimates $(95 \% \mathrm{CI})$ & $\mathrm{I}^{2}$ tests with p-value \\
\hline Study designs & Case control & $50.02 \%(22.78,77.26 \%)$ & $96.1 \%, \mathrm{P}=0.000$ \\
& Cohort & $27.36 \%(19.23,35.48 \%)$ & $91.6 \%, \mathrm{P}=0.000$ \\
& Cross-sectional & $38.66 \%(19.82,57.51 \%)$ & $97.4 \%, \mathrm{P}=0.000$ \\
\multirow{2}{*}{ Treatment modalities } & Three treatments & $45.46 \%(27.23,63.70 \%)$ & $97.5 \%, \mathrm{P}=0.000$ \\
& Less three & $26.5 \%(20.29,32.71 . \%)$ & $87.3 \%, \mathrm{P}=0.000$ \\
\multirow{2}{*}{ Measuring tools } & treatments & & \\
& IIEF & $40.50 \%(25.42,55.58)$ & $97.5 \%, \mathrm{P}=0.000$ \\
& BMSFI & $31.49 \%(22.48,40.50)$ & $71.9 \%, \mathrm{P}=0.06$ \\
& Others & $24.48 \%(18.30,30.66)$ & $74.7 \%, \mathrm{P}=0.008$ \\
\hline
\end{tabular}

Others= International Index of Erectile Function and Brief Sexual Function Inventory, European Organization for Research and Treatment of Cancer (EORTC QLQ-PR25), nine-item generic questionnaire, adverse health outcomes

To estimate the pooled prevalence of erectile dysfunction a random-effect model was employed to this analysis. The study design, publication year, sample size and response rate were inspected using multivariate meta-regression model whether they were associated with the heterogeneity. As the model shows, none of them were statistically significant (Table 3). 
Table 3. Related factors with heterogeneity of erectile dysfunction prevalence among testicular cancer survivor's in the current meta-analysis, 2020.

\begin{tabular}{lcc}
\hline Variables & Coefficient & P-value \\
\hline Study design & $-18.72(-57.72,20.27)$ & 0.300 \\
Publication year & $.533(-2.94,4.01)$ & 0.733 \\
Sample size & $-.011(-.035, .013)$ & 0.340 \\
Response rate & $.092(-.879,1.063)$ & 0.833 \\
\hline
\end{tabular}

\subsubsection{Publication bias.}

To assess publication bias, the funnel plot and Egger's test were conducted in the meta-analysis (Figure 3). The result of the Egger test was also statistically significant with Bo $=3.33$ and $p<$ 0.001. To see publication bias further, trim fill analysis was done, and unpublished studies were not found.

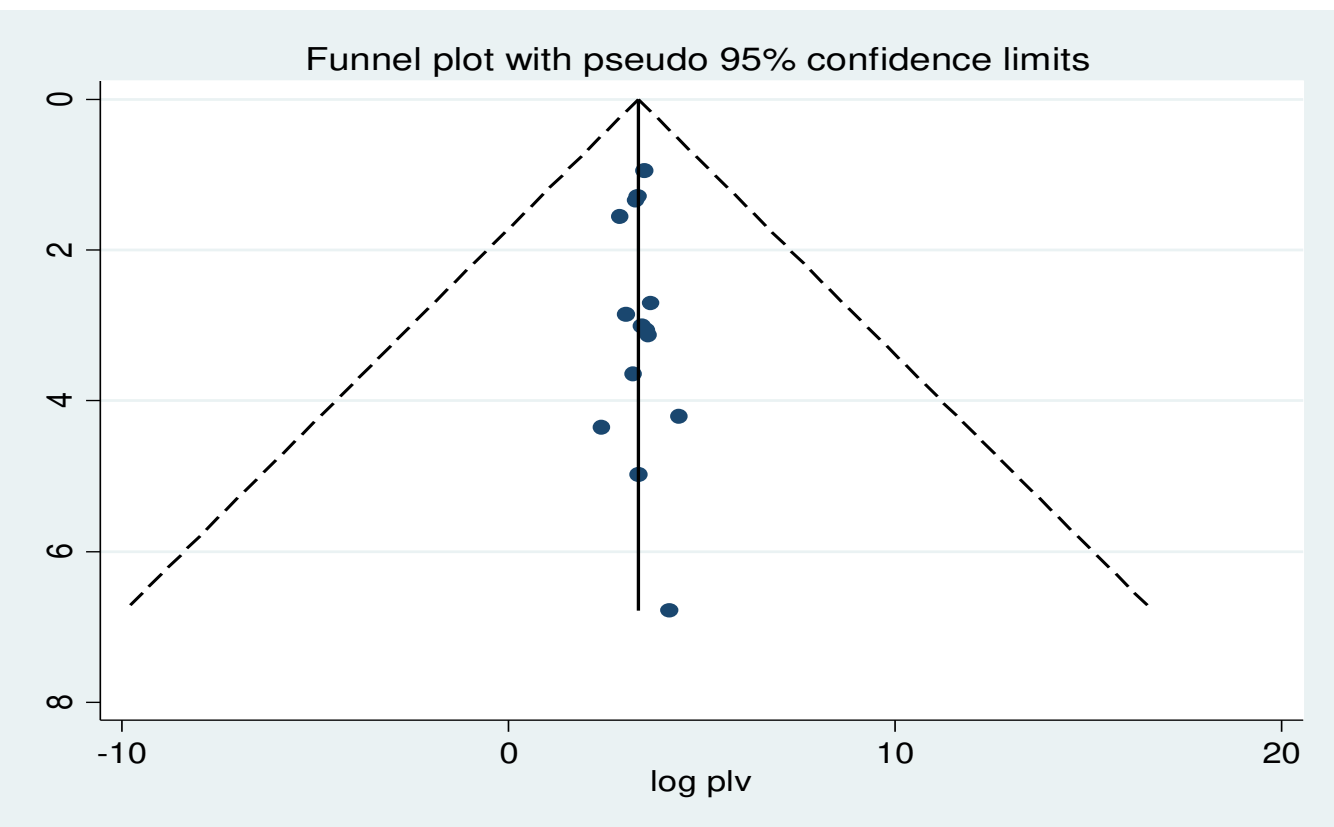


Figure 3. Funnel plot for publication bias, logprop or $\operatorname{lnp}$ (log of proportion) represented in the $\mathrm{X}$-Axis and standard error of $\log$ proportion in the $\mathrm{Y}$-Axis.

\subsubsection{Sensitivity analysis}

Among all fourteen reviewed studies in the current analysis, no study had shown an impact on the overall estimation (Figure 4).

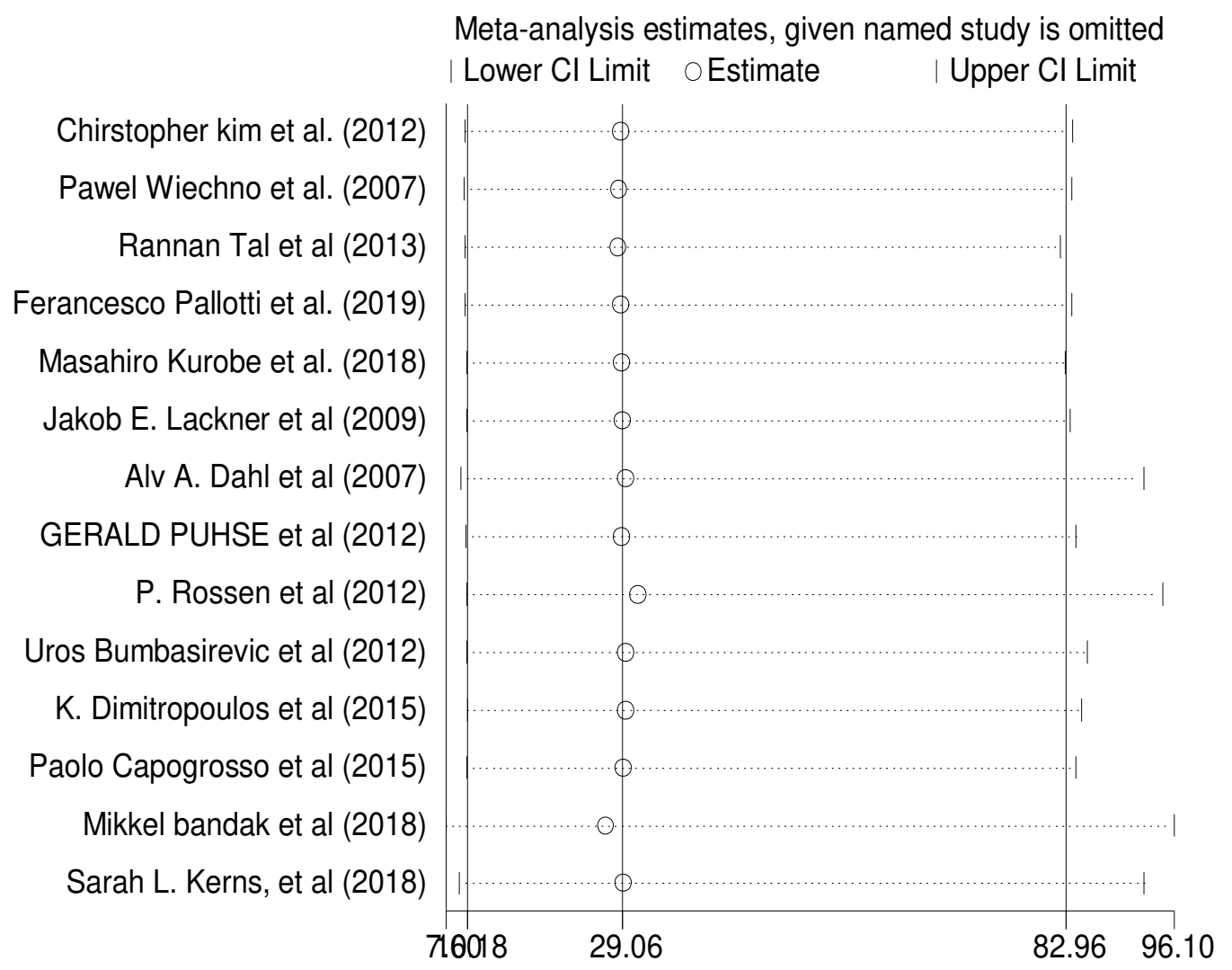

Figure 4. The sensitivity analysis showed the pooled prevalence when the studies omitted step by step. 


\section{Discussion}

This meta-analysis showed that the pooled prevalence of erectile dysfunction among testicular cancer survivors was $34.6 \%$. This pooled prevalence of erectile dysfunction among testicular cancer survivors is higher than studies conducted in the London 8\% [35], China 16.9\% [38], and Netherlands $11.5 \%$ [39].

This discrepancy may be due to the age difference of study participants, the time of study, study design, types of testicular cancer (unilateral or bilateral), and treatment modalities. A study conducted in London included only young men (16-39 years old). But the current study includes all age groups and older age increases the prevalence of erectile dysfunction $[5,40]$. In the case of a study conducted in China, the study was only included case control studies and patients experienced unilateral orchiectomy nevertheless this systematic review and meta-analysis study includes any primary studies that clearly reported the prevalence of erectile dysfunction among testicular cancer survivors and patients underwent both unilateral orchiectomy and/or bilateral orchiectomy. Evidence supported that bilateral orchiectomy had an associated with the prevalence of erectile dysfunction in testicular cancer survivors due to complete cessation or very low production of testosterone [31]. And, the discrepancy between a study conducted in Netherland and this study may be due to the time variation and the study design that included in the systematic review and meta-analysis. A study done in Netherland was conducted before two decades and included only cohort studies conducted from 1975 to 2000. But the current study includes all primary studies conducted from 20001 to 2020 . In the last two decades the survival rate of testicular cancer is highly increased, this in turn increases the prevalence of erectile dysfunction in testicular cancer survivors due to long effects of testicular cancer complications [30]. 
Subgroup analysis piercing out that the prevalence of erectile dysfunction was $45.46 \%$ among those who were treated with three treatment modalities and the prevalence of erectile dysfunction was $26.5 \%$ among those testicular cancer survivors treated with less than three treatment modalities. This indicated that substantial heterogeneity of the prevalence of erectile dysfunction across treatment modalities. This variance might be due to the synergistic effect of three treatment modalities on erectile function. Evidence showed that Chemotherapy, surgery, and radiation can all cause sexual antagonistic effect by causing neuropathy, nerve disconnected and interruption of normal blood supply to maintain erection and vasculogenic respectively [3, 5]. The combination of these treatments has much more negative effect on erection than treatments less than this combination.

The heterogeneity of erectile dysfunction prevalence is also seen across different measurement tools. The prevalence of ED in the studies used IIEF as a criterion tool was higher than other studies which were used BMSFI and others criterion tools. This might be due to the different cut points to determine erectile dysfunction among tools. This finding is supported by a study conducted in China [38].

The result of this study will have its own limitations. In doing this research we only considered articles that reported their findings using English language. The other limitation is that the severity of ED was not measured and described because of lack of sufficient information from the studies. Subgroup analysis using age was not done due to lack of data in the included studies. All the articles included to this study were conducted in Europe or America. Hence, the results may not be inferred to patients in Asia or Africa. 


\section{Conclusion}

There was a high prevalence of erectile dysfunction in testicular cancer survivors. It had also causes of heterogeneity in terms of treatment modalities, study designs and measuring tools. Hence, there is a trepidation for responsible bodies to prevent the high level of this complication.

\section{List of abbreviations}

BMSFI= Brief Male Sexual Function Inventory

$\mathrm{ED}=$ Erectile dysfunction

IIEF= International Index of Erectile Function

JBI=Joanna Briggs Institute

$\mathrm{TC}=$ Testicular Cancer

\section{Declarations}

\section{Ethics approval and consent to participate}

Not applicable

\section{Consent for publication}

Not applicable

\section{Availability of data and materials}

Not applicable

\section{Competing interest}

The authors declare no conflict of interest.

\section{Funding}

This research did not receive any specific grant from funding agencies in the public, commercial, or not-for-profit sectors. 


\section{Authors' contribution}

All authors listed have significantly contributed to the development and the writing of this article.

Acknowledgements

Not applicable 


\section{Reference}

1. Kim C, McGlynn KA, McCorkle R, Li Y, Erickson RL, Ma S, Niebuhr DW, Zhang G, Zhang Y, Bai Y: Sexual functioning among testicular cancer survivors: a case-control study in the US. Journal of psychosomatic research 2012, 73(1):68-73.

2. Pishgar F, Haj-Mirzaian A, Ebrahimi H, Saeedi Moghaddam S, Mohajer B, Nowroozi MR, Ayati M, Farzadfar F, Fitzmaurice C, Amini E: Global, regional and national burden of testicular cancer, 1990-2016: results from the Global Burden of Disease Study 2016. BJU international 2019, 124(3):386-394.

3. Tal R, Stember DS, Logmanieh N, Narus J, Mulhall JP: Erectile dysfunction in men treated for testicular cancer. BJU international 2014, 113(6):907-910.

4. Pühse G, Wachsmuth JU, Kemper S, Husstedt IW, Evers S, Kliesch S: Chronic pain has a negative impact on sexuality in testis cancer survivors. Journal of andrology 2012, 33(5):886-893.

5. Voznesensky M, Annam K, Kreder KJ: Understanding and managing erectile dysfunction in patients treated for cancer. Journal of oncology practice 2016, 12(4):297-304.

6. Dimitropoulos K, Karatzas A, Papandreou C, Daliani D, Zachos I, Pisters L, Tzortzis V: Sexual dysfunction in testicular cancer patients subjected to post-chemotherapy retroperitoneal lymph node dissection: a focus beyond ejaculation disorders. Andrologia 2016, 48(4):425-430.

7. Yafi FA, Jenkins L, Albersen M, Corona G, Isidori AM, Goldfarb S, Maggi M, Nelson CJ, Parish S, Salonia A: Erectile dysfunction. Nature reviews Disease primers 2016, 2(1):120. 
8. Tuinman MA, Fleer J, Sleijfer DT, Hoekstra HJ, Hoekstra-Weebers JE: Marital and sexual satisfaction in testicular cancer survivors and their spouses. Supportive Care in Cancer 2005, 13(7):540-548.

9. Varela VS, Zhou ES, Bober SL: Management of sexual problems in cancer patients and survivors. Curr Probl Cancer 2013, 37(6):319-352.

10. Pallotti F, Petrozzi A, Cargnelutti F, Radicioni AF, Lenzi A, Paoli D, Lombardo F: Longterm follow up of the erectile function of testicular cancer survivors. Frontiers in endocrinology 2019, 10:196.

11. Jankowska M: Sexual functioning of testicular cancer survivors and their partners-A review of literature. Reports of Practical Oncology \& Radiotherapy 2012, 17(1):54-62.

12. Rossen P, Pedersen AF, Zachariae R, von der Maase H: Sexuality and body image in long-term survivors of testicular cancer. European journal of cancer 2012, 48(4):571578.

13. Chovanec M, Vasilkova L, Petrikova L, Obertova J, Palacka P, Rejlekova K, Sycova-Mila Z, Kalavska K, Svetlovska D, Mladosievicova B: Long-term sexual functioning in germcell tumor survivors. BMC cancer 2020, 20(1):1-10.

14. Eberhard J, Ståhl O, Cohn-Cedermark G, Cavallin-Ståhl E, Giwercman Y, Rylander L, Eberhard-Gran M, Kvist U, Fugl-Meyer KS, Giwercman A: Sexual function in men treated for testicular cancer. The journal of sexual medicine 2009, 6(7):1979-1989.

15. Bandak M, Lauritsen J, Johansen C, Kreiberg M, Skøtt JW, Agerbaek M, Holm NV, Daugaard G: Sexual function in a nationwide cohort of $\mathbf{2 , 2 6 0}$ survivors of testicular cancer after 17 years of followup. The Journal of urology 2018, 200(4):794-800. 
16. Schepisi G, De Padova S, De Lisi D, Casadei C, Meggiolaro E, Ruffilli F, Rosti G, Lolli C, Ravaglia G, Conteduca V: Psychosocial issues in long-term survivors of testicular cancer. Frontiers in Endocrinology 2019, 10:113.

17. Moher D, Liberati A, Tetzlaff J, Altman DG, Group P: Preferred reporting items for systematic reviews and meta-analyses: the PRISMA statement. PLoS med 2009, 6(7):e1000097.

18. Jordan Z, Lockwood C, Munn Z, Aromataris E: The updated Joanna Briggs Institute model of evidence-based healthcare. International journal of evidence-based healthcare 2019, 17(1):58-71.

19. Noordzij M, Dekker FW, Zoccali C, Jager KJ: Measures of disease frequency: prevalence and incidence. Nephron Clinical Practice 2010, 115(1):c17-c20.

20. Stang A: Critical evaluation of the Newcastle-Ottawa scale for the assessment of the quality of nonrandomized studies in meta-analyses. European journal of epidemiology 2010, 25(9):603-605.

21. Egger M, Smith GD, Schneider M, Minder C: Bias in meta-analysis detected by a simple, graphical test. Bmj 1997, 315(7109):629-634.

22. Ioannidis JP: Interpretation of tests of heterogeneity and bias in meta-analysis. Journal of evaluation in clinical practice 2008, 14(5):951-957.

23. Higgins JP, Thompson SG: Quantifying heterogeneity in a meta-analysis. Statistics in medicine 2002, 21(11):1539-1558.

24. Borenstein M, Hedges LV, Higgins JP, Rothstein HR: A basic introduction to fixed-effect and random-effects models for meta-analysis. Research synthesis methods 2010, 1(2):97-111. 
25. Wiechno P, Demkow T, Kubiak K, Sadowska M, Kamińska J: The quality of life and hormonal disturbances in testicular cancer survivors in Cisplatin era. European urology 2007, 52(5):1448-1455.

26. Kurobe M, Kawai K, Suetomi T, Iwamoto T, Waku N, Kawahara T, Kojima T, Joraku A, Miyazaki J, Nishiyama H: High prevalence of hypogonadism determined by serum free testosterone level in Japanese testicular cancer survivors. International Journal of Urology 2018, 25(5):457-462.

27. Lackner JE, Koller A, Schatzl G, Marberger M, Kratzik C: Androgen deficiency symptoms in testicular cancer survivors are associated with sexual problems but not with serum testosterone or therapy. Urology 2009, 74(4):825-829.

28. Dahl AA, Bremnes R, Dahl O, Klepp O, Wist E, Fosså SD: Is the sexual function compromised in long-term testicular cancer survivors? European urology 2007, 52(5):1438-1447.

29. Bumbasirevic U, Bojanic N, Pekmezovic T, Janjic A, Janicic A, Milojevic B, Tulic C: Health-related quality of life, depression, and sexual function in testicular cancer survivors in a developing country: a Serbian experience. Supportive care in cancer 2013, 21(3):757-763.

30. Capogrosso P, Boeri L, Ferrari M, Ventimiglia E, La Croce G, Capitanio U, Briganti A, Damiano R, Montorsi F, Salonia A: Long-term recovery of normal sexual function in testicular cancer survivors. Asian journal of andrology 2016, 18(1):85.

31. Bandak M, Lauritsen J, Johansen C, Kreiberg M, Skøtt JW, Agerbaek M, Holm NV, Daugaard G: Sexual function and quality of life in a national cohort of survivors of bilateral testicular cancer. European urology focus 2018. 
32. Lackner J, Schatzl G, Koller A, Mazal P, Waldhoer T, Marberger M, Kratzik C: Treatment of testicular cancer: influence on pituitary-gonadal axis and sexual function. Urology 2005, 66(2):402-406.

33. Kerns SL, Fung C, Monahan PO, Ardeshir-Rouhani-Fard S, Zaid MIA, Williams AM, Stump TE, Sesso HD, Feldman DR, Hamilton RJ: Cumulative burden of morbidity among testicular cancer survivors after standard cisplatin-based chemotherapy: a multi-institutional study. Journal of Clinical Oncology 2018, 36(15):1505.

34. Hartmann J, Albrecht C, Schmoll H, Kuczyk M, Kollmannsberger C, Bokemeyer C: Longterm effects on sexual function and fertility after treatment of testicular cancer. British journal of cancer 1999, 80(5):801-807.

35. Ljungman L, Eriksson L, Flynn K, Gorman J, Ståhl O, Weinfurt K, Wiklander M, Lampic C, Wettergren L: Sexual dysfunction and reproductive concerns in young men diagnosed with testicular cancer: an observational study. The Journal of Sexual Medicine 2019, 16(7):1049-1059.

36. Agrawal V, Dinh Jr PC, Fung C, Monahan PO, Althouse SK, Norton K, Cary C, Einhorn L, Fossa SD, Adra N: Adverse Health Outcomes Among US Testicular Cancer Survivors After Cisplatin-Based Chemotherapy vs Surgical Management. JNCI Cancer Spectrum 2020, 4(2):pkz079.

37. van Iersel L, Li Z, Chemaitilly W, Schover LR, Ness KK, Hudson MM, Klosky JL: Erectile dysfunction in male survivors of childhood cancer. JAMA oncology 2018, 4(11):1613-1616.

38. Xiong J, Zhang J, Cai Z, Ma C, Li H: Erectile dysfunction in testicular cancer survivors: a meta-analysis of case-control studies. Archives of Medical Science, 16(1). 
39. Jonker-Pool G, Van de Wiel HB, Hoekstra HJ, Sleijfer DT, Van Driel MF, Van Basten JP, Koops HS: Sexual functioning after treatment for testicular cancer-review and meta-analysis of 36 empirical studies between 1975-2000. Archives of Sexual Behavior 2001, 30(1):55-74.

40. Gareri P, Castagna A, Francomano D, Cerminara G, De Fazio P: Erectile dysfunction in the elderly: an old widespread issue with novel treatment perspectives. International journal of endocrinology 2014, 2014. 


\section{Figures}
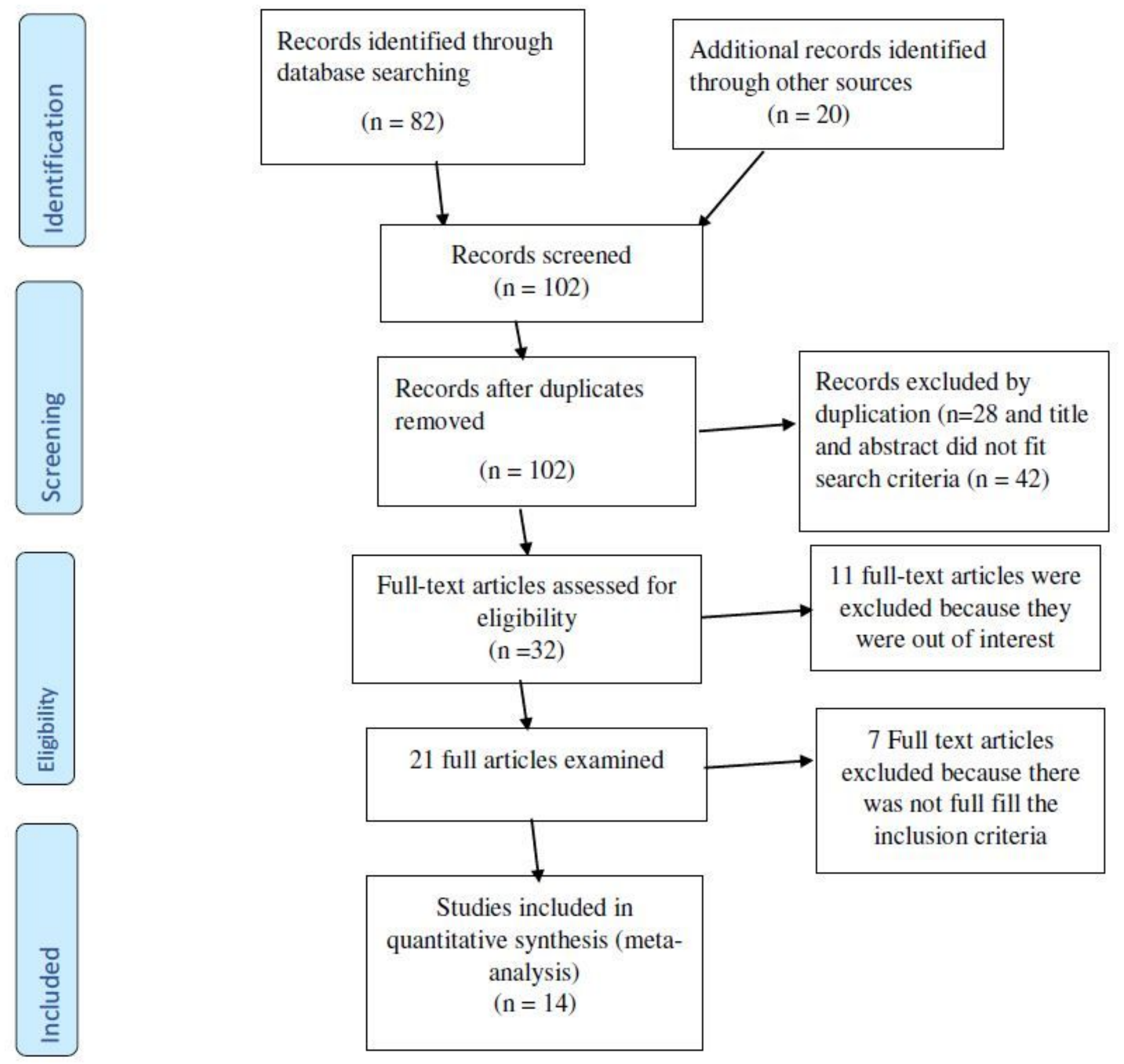

Figure 1

Flowchart to a selection of studies for a systematic review and meta-analysis of the prevalence of erectile dysfunction, 2020. 
Study

ID
ES $(95 \% \mathrm{Cl})$

Weight

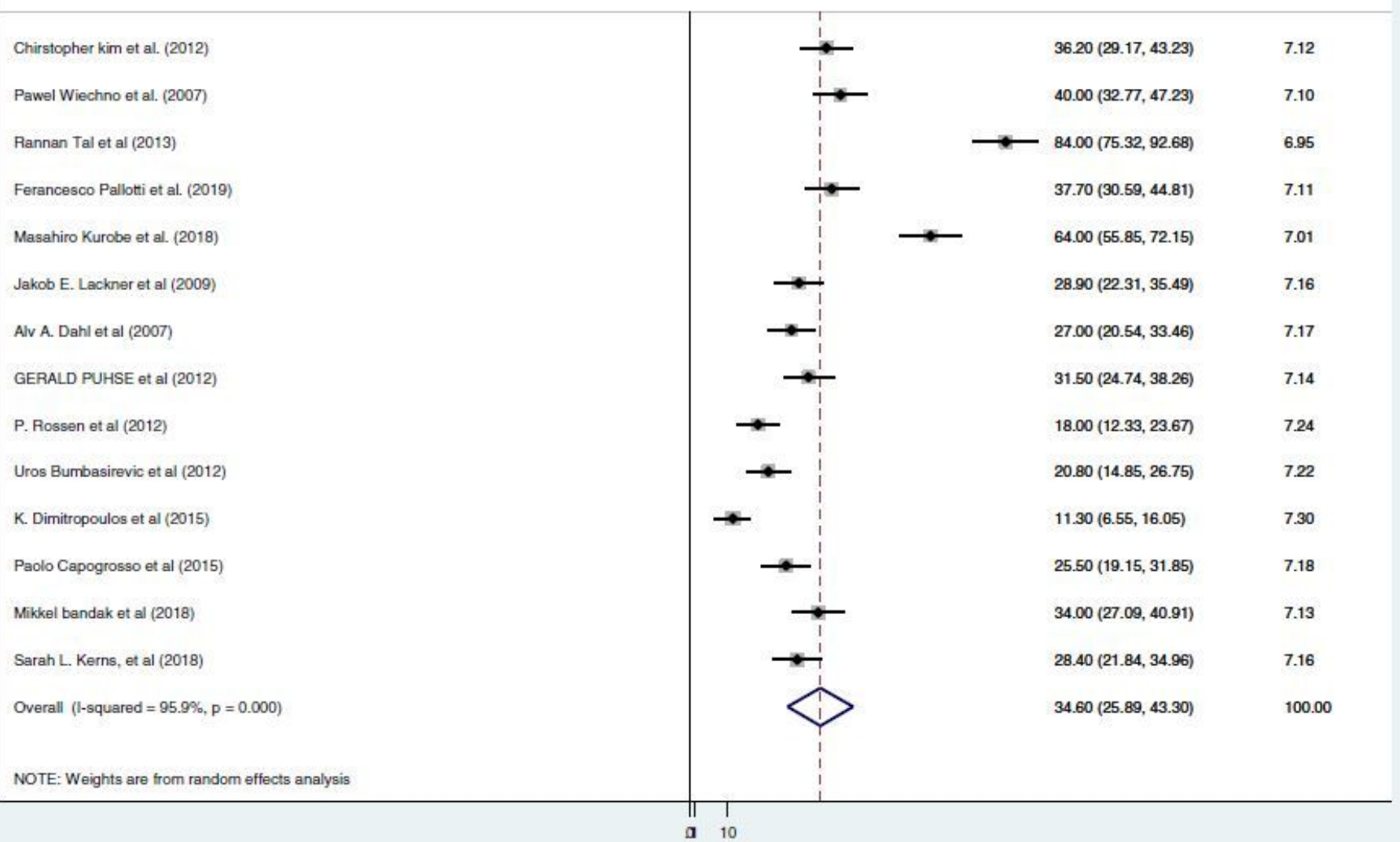

\section{Figure 2}

Pooled prevalence of erectile dysfunction among testicular cancer survivors, $2020(n=14)$. 


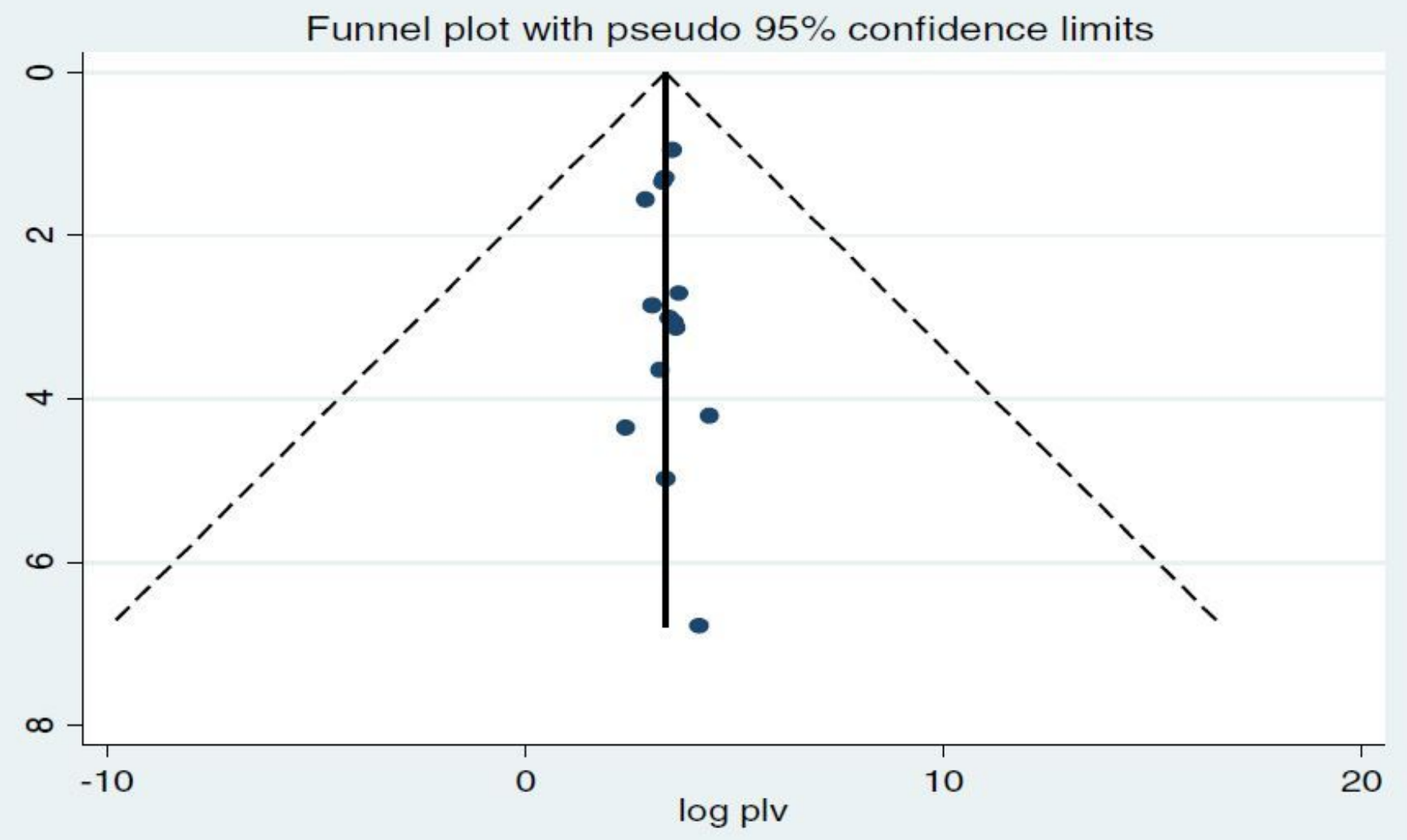

Figure 3

Funnel plot for publication bias, logprop or Inp (log of proportion) represented in the X-Axis and standard error of log proportion in the Y-Axis. 
Meta-analysis estimates, given named study is omitted | Lower Cl Limit oEstimate | Upper Cl Limit

Chirstopher kim et al. (2012)

Pawel Wiechno et al. (2007)

Rannan Tal et al (2013)

Ferancesco Pallotti et al. (2019)

Masahiro Kurobe et al. (2018)

Jakob E. Lackner et al (2009)

Alv A. Dahl et al (2007)

GERALD PUHSE et al (2012)

P. Rossen et al (2012)

Uros Bumbasirevic et al (2012)

K. Dimitropoulos et al (2015)

Paolo Capogrosso et al (2015)

Mikkel bandak et al (2018)

Sarah L. Kerns, et al (2018)

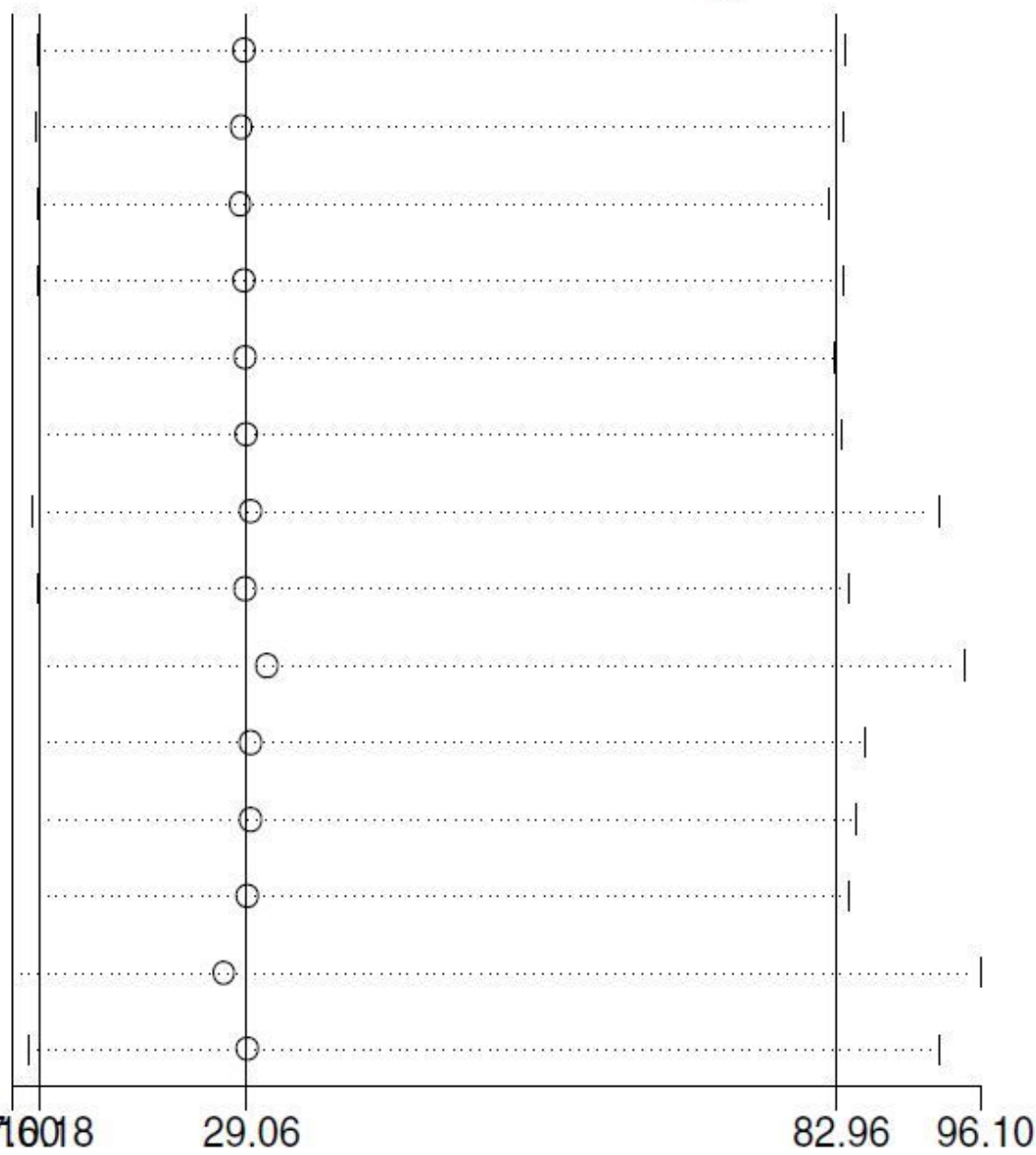

\section{Figure 4}

The sensitivity analysis showed the pooled prevalence when the studies omitted step by step. 